\title{
Descripción clínico-morfológica y factores pronósticos en carcinomas de apéndice cecal: Estudio de cohorte*
}

\author{
Drs. OSCAR TAPIA E..$^{1,3}$, CARLOS MANTEROLA D. ${ }^{2,4}$, MIGUEL VILLASECA H..$^{1,3}$, \\ JUAN CARLOS ARAYA O. ${ }^{1,3}$, PABLO GUZMÁN G. ${ }^{1,3}$, JUAN CARLOS ROA S. ${ }^{1,3}$

\begin{abstract}
Departamento de Anatomía Patológica. Facultad de Medicina. Universidad de La Frontera. Departamento de Cirugía y Traumatología, Facultad de Medicina. Universidad de La Frontera. BIOREN (Scientifical and Technological Bioresources Nucleus). Temuco, Chile.
\end{abstract} \\ 4 CIGES (Training, Investigation and Evidence for Based Health Medicine), Universidad de La Frontera.
}

\begin{abstract}
Appendiceal malignant tumors. Review of 84 cases

Background: Malignant appendiceal tumors account for only $0.5 \%$ of all gastrointestinal tumors and there are usually diagnosed during the pathological study of excised appendices. Aim: To assess the factors influencing the survival of patients with appendiceal carcinomas. Material and Methods: Retrospective review of records of all primary appendiceal carcinomas diagnosed in the pathology unit of a regional hospital between 1993 and 2008. The surgical procedure, clinical history, morphological features of tumor and survival were recorded. Results: Eighty four patients aged $32 \pm 15$ years (44 women) with appendiceal carcinoma were identified. In $93 \%$ of patients, the clinical diagnosis was acute appendicitis. Sixty one patients $(73 \%)$ had a carcinoid tumor, 20 (24\%) an adenocarcinoma and three (3\%), an adenocarcinoid tumor. Preoperative suspicion of a malignant tumor, level of tumor infiltration, pathological type, involvement of surgical borders, tumor size over $2 \mathrm{~cm}$ in the case of carcinoid tumors and the degree of differentiation of adenocarcinomas, had prognostic value. For adenocarcinomas, performing a right hemicolectomy significantly improved survival. Conclusions: Carcinoid tumors are the most common malignant tumor of the appendix. A right hemicolectomy may improve the survival of patients with appendiceal adenocarcinomas.
\end{abstract}

Key words: Appendix, carcinoid tumor, adenocarcinoma.

\section{Resumen}

Introducción: Los tumores malignos del apéndice cecal representan tan sólo el 0,5\% de todas las neoplasias gastrointestinales. Su diagnóstico generalmente no es sospechado por el clínico, siendo su diagnóstico un hallazgo durante el examen de la pieza operatoria. Algunos de estos tumores presentan buena supervivencia con la sola apendicetomía, aunque en ocasiones es necesario realizar tratamientos complementarios. El objetivo de este estudio es determinar la asociación entre variables clínicas y morfológicas con la supervivencia de pacientes con carcinomas del apéndice cecal y analizar el tratamiento quirúrgico realizado. Material y Método: Cohorte retrospectiva de 84 pacientes con carcinomas apendiculares diagnosticados en el Hospital Hernán Henríquez Aravena de Temuco entre los años 1993-2008. Se estudiaron variables clínico-morfológicas, el tratamiento quirúrgico realizado y la supervivencia para estos pacientes. Se utilizó

*Recibido el 3 de Diciembre de 2009 y aceptado para publicación el 19 de Enero de 2010.

Financiado en parte por la Dirección de investigación de la Universidad de La Frontera.

Correspondencia: Dr. Juan Carlos Roa S. Manuel Montt 112. Código Postal 478-1176. Temuco, Chile.

E-mail: jcroa@ufro.cl 
estadística descriptiva y analítica, aplicando Chi-cuadrado y test exacto de Fisher para variables categóricas y T-Student para variables continuas; y prueba Log-rank (Mantel-Cox) para comparación de curvas de supervivencia con un IC de 95\%. Resultados: Del total de apendicectomías (18.563) se verificaron 84 casos (0,45\%); 48\% hombres y 52\% mujeres con una edad promedio de 31,9 \pm 15 años. En el 93\% de los casos el diagnóstico clínico fue apendicitis aguda. Correspondieron a carcinoide, adenocarcinoma y adenocarcinoide en $61(73 \%), 20(24 \%)$ y $3(3 \%)$ casos respectivamente. En el análisis bivariado las siguientes variables mostraron asociación pronóstica estadísticamente significativa: sospecha pre-operatoria $(\mathrm{p}=0,009)$, nivel de infiltración tumoral $(\mathrm{p}=0,04)$, tipo histológico $(\mathrm{p}=0,009)$, compromiso de bordes quirúrgicos $(\mathrm{p}<0,001)$, tamaño tumoral $>2 \mathrm{~cm}$ en tumores carcinoides $(\mathrm{p}=0,001)$ y grado de diferenciación en adenocarcinomas $(\mathrm{p}$ $<0,001)$. La realización de hemicolectomía derecha complementaria demostró diferencias estadísticamente significativas en el subgrupo de pacientes con adenocarcinomas apendiculares $(p=0,04)$. Conclusiones: Los tumores apendiculares son neoplasias poco frecuentes, generalmente no sospechados pre-operatoriamente y diagnosticados durante el examen anátomo-patológico, siendo tumores carcinoides el tipo histológico más frecuente en apéndice cecal. Está demostrado el beneficio de la hemicolectomía derecha en adenocarcinomas, no demostrándose su beneficio en tumores carcinoides.

Palabras clave: Neoplasia apendicular [MeSH], tumor carcinoide [MeSH], pronóstico [MeSH], factores de pronóstico [MeSH].

\section{Introducción}

Los tumores malignos del apéndice cecal son un grupo de neoplasias poco frecuentes, representando tan sólo el 0,5\% de todas las neoplasias gastrointestinales ${ }^{1-6}$. Entre el 50\%-75\% corresponden a tumores con diferenciación neuroendocrina, constituyendo la localización apendicular aproximadamente el $19 \%$ de estos carcinomas ${ }^{2,3}$. Tumores carcinoides y adenocarcinomas apendiculares se encuentran en aproximadamente el $1 \%$ y $0,1 \%$ de las apendicectomías realizadas ${ }^{4-7}$.

Habitualmente el diagnóstico no es sospechado en el pre-operatorio, siendo estos pacientes en su mayoría intervenidos quirúrgicamente con diagnóstico clínico de apendicitis aguda, por lo que el diagnóstico se plantea recién con el examen de la pieza operatoria ${ }^{1,3,5-9}$. Otro elemento descrito es el pronóstico variable entre los distintos tipos histológicos, condición que determina además la necesidad de efectuar tratamientos complementarios tales como hemicolectomía derecha con criterio oncológico asociado o no a quimioterapia; considerando conjuntamente para ello el tamaño y localización tumoral, nivel de infiltración en la pared apendicular y compromiso de bordes de sección. Si bien la hemicolectomía derecha ha demostrado mejorar la supervivencia en pacientes con adenocarcinomas apendiculares su beneficio en los carcinomas neuroendocrinos no ha sido bien documentado, lo que dificulta aún más evaluar su impacto en la supervivencia de estos pacientes, dado la heterogeneidad clínica y metodológica de los estudios publicados ${ }^{6-10,12,14,16,17}$.

A pesar de estas consideraciones, tumores de diámetro menor a $1 \mathrm{~cm}$ confinados al apéndice cecal y en los que sólo se realiza apendicectomía presentan supervivencia superior a $95 \%$ a 5 años, lo que hace más discutible la necesidad de efectuar cirugía u otras terapias complementarias ${ }^{5-8}$.

El objetivo de este estudio es describir aspectos clínicos y morfológicos de pacientes con diagnóstico de carcinoma apendicular y determinar posibles asociaciones pronósticas de estas variables con la supervivencia alejada.

\section{Material y Método}

Diseño del estudio: Cohorte retrospectiva.

Criterios de inclusión: Se incluyeron todos los casos de carcinomas apendiculares primarios diagnosticados en la Unidad de Anatomía Patológica del Hospital Dr. Hernán Henríquez Aravena de Temuco, desde Enero de 1993 hasta Diciembre de 2008. No se consideraron criterios de exclusión.

Protocolo de procesamiento: En este período, el procesamiento de las piezas quirúrgicas de apendicectomía con diagnóstico anatomo-patológico tumoral fue realizado mediante mapeo completo de la pieza operatoria con identificación de borde quirúrgico proximal y circunferencial con el objeto de determinar el máximo nivel de infiltración de la lesión y compromiso de bordes quirúrgicos.

Clasificación histológica: Se utilizó la clasificación histológica de la Organización Mundial de la Salud (OMS) para tumores apendiculares ${ }^{9}$.

Nivel de infiltración: Definiéndose para cada uno de ellos el nivel de infiltración máximo en la pared apendicular, determinado por el mapeo completo de la pieza quirúrgica.

Localización macroscópica: Se consideró tercio proximal (base), medio y distal (punta). Las 
DESCRIPCIÓN CLÍNICO-MORFOLÓGICA Y FACTORES PRONÓSTICOS EN CARCINOMAS DE APÉNDICE CECAL: ...

lesiones identificadas fueron adscritas a los tercios correspondientes, en aquellos casos que existía compromiso de más de un tercio se revisaron archivos fotográficos y digitales para determinar la probable localización primaria del tumor.

Variables estudiadas: La variable resultado o independiente fue "supervivencia", determinada en meses desde el postoperatorio; las variables asociadas estudiadas fueron las siguientes: Edad, género, localización, tamaño tumoral, tipo histológico, grado histológico de diferenciación y nivel de infiltración tumoral. Se consideró además el impacto del tratamiento complementario que recibieron algunos pacientes.

Seguimiento: Se tuvo seguimiento desde el momento del diagnóstico a través de: controles clínicos y bases de datos de certificación de defunción del Departamento de Informática de la Dirección del Servicio de Salud Araucanía Sur. Se consideraron como "vivos" aquellos casos de defunción en los que el rol del cáncer apendicular no figuraba como causa de muerte.

Plan de análisis: Utilizando los paquetes estadísticos Epi-info 6.0 y Stata 9.0, se realizó un análisis exploratorio de los datos y posteriormente se aplicó estadística descriptiva con cálculo de promedios y desviaciones estándar, medianas y valores extremos para variables continuas; cálculo de porcentajes para variables categóricas; y curvas de supervivencia actuarial de Kaplan-Meier. Luego, se aplicó estadística analítica, utilizando t-test y análisis de la varianza (ANOVA) para variables continuas, Chi cuadrado de Pearson y exacto de Fisher para variables categóricas; y prueba Log-rank (Mantel-Cox) para comparación de curvas de supervivencia con un IC de 95\%.

\section{Resultados}

Se realizaron en el período analizado 18.563 apendicetomías, diagnosticándose 84 casos de carcinomas apendiculares $(0,45 \%)$; correspondiendo 61 casos $(73 \%)$ a carcinoides, 20 casos $(24 \%)$ adenocarcinomas y 3 casos (3\%) adenocarcinoides (Tabla 1).

El 52\% de los sujetos en estudio eran mujeres. La edad promedio del grupo fue 31,9 $\pm 19,5$ años, siendo el promedio de edad para carcinoides, adenocarcinomas y adenocarcinoides $24,7 \pm 15$ años, $53,2 \pm 16$ años y $39,3 \pm 16$ años respectivamente $(\mathrm{p}$ $<0,05)$ (Tabla 1).

Tabla 1. Distribución de algunas variables clínicas y morfológicas por tipo histológico

\begin{tabular}{lccc}
\hline & $\begin{array}{c}\text { Carcinoide } \\
(\mathbf{n = 6 1 )}\end{array}$ & $\begin{array}{c}\text { Adenocarcinoma } \\
(\mathbf{n = 2 0})\end{array}$ & $\begin{array}{c}\text { Adenocarcinoide } \\
(\mathbf{n}=\mathbf{3})\end{array}$ \\
\hline Género & $54 \%(33)$ & $45 \%(9)$ & $67 \%(2)$ \\
Femenino & $46 \%(28)$ & $55 \%(11)$ & $33 \%(1)$ \\
Masculino & $24,7 \pm 15$ años & $53,2 \pm 16$ años & $39,3 \pm 16$ años \\
Edad (Promedio) & $1 / 61$ & $5 / 20$ & $0 / 3$ \\
Fallecidos/Total & 80 meses & 83 meses & 106 meses \\
Promedio seguimiento (meses) & $97 \%$ & $75 \%$ & $100 \%$ \\
Supervivencia a 5 años & & & $100 \%(3)$ \\
Sospecha pre-operatoria tumoral & $98 \%(60)$ & $75 \%(15)$ & - \\
No & $2 \%(1)$ & $25 \%(5)$ & $100 \%(3)$ \\
Sí & $98 \%(60)$ & $80 \%(16)$ & - \\
Compromiso bordes quirúrgicos & $2 \%(1)$ & $20 \%(4)$ & - \\
No & & & - \\
Sí & $2 \%(1)$ & $15 \%(3)$ & - \\
Infiltración & $2 \%(1)$ & $10 \%(2)$ & $33 \%(1)$ \\
Mucosa & $31 \%(19)$ & $5 \%(1)$ & $67 \%(2)$ \\
Submucosa & $34 \%(21)$ & $30 \%(6)$ & - \\
Muscular propia & $10 \%(6)$ & $15 \%(3)$ & - \\
Subserosa & $21 \%(13)$ & $40 \%(8)$ & $100 \%(3)$ \\
Serosa & $8 \%(5)$ & $60 \%(12)$ & - \\
Mesoapéndice & $92 \%(56)$ & & \\
Hemicolectomía derecha & & & - \\
Sí & & & \\
No & & & \\
\hline
\end{tabular}




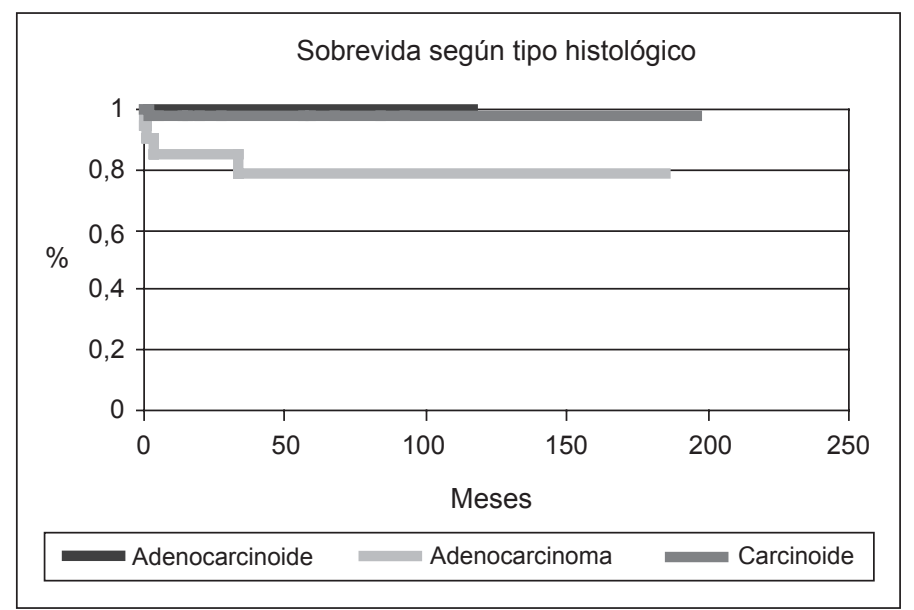

Figura 1.

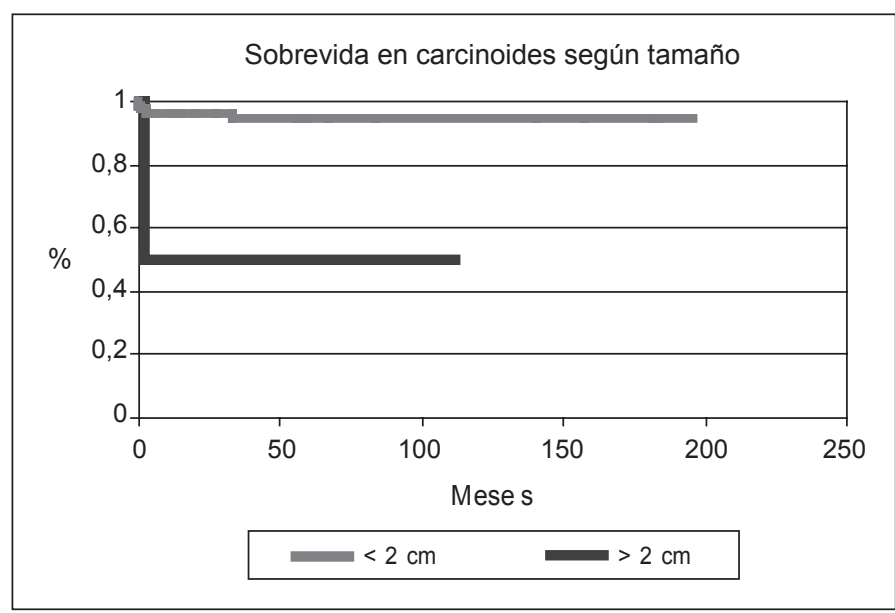

Figura 2.

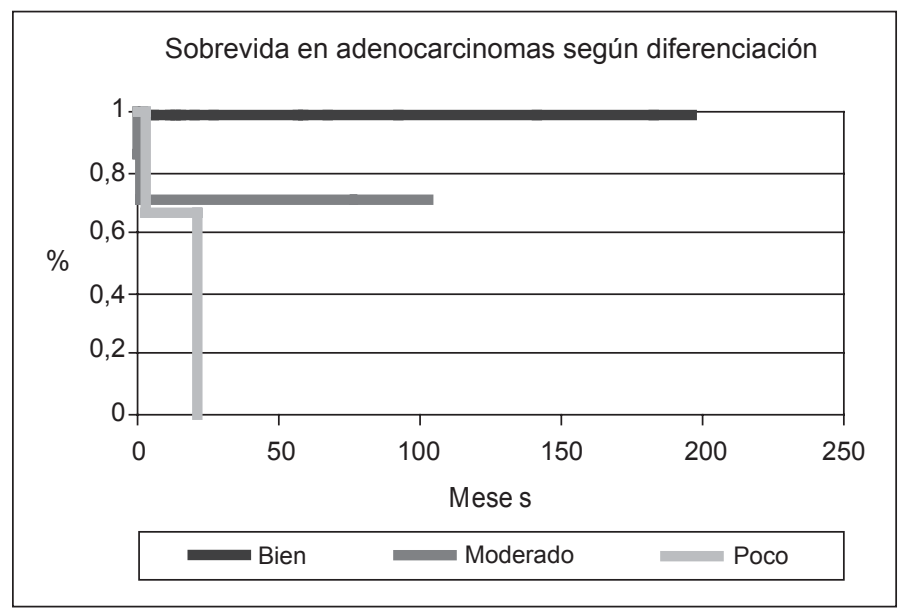

Figura 3.
En el 93\% de los casos el diagnóstico clínico pre-operatorio correspondió a patología inflamatoria, siendo sospechada la lesión tumoral en el 7\% de los pacientes; la sospecha pre-operatoria fue mayor para adenocarcinomas que carcinoides $(25 \%$ y $2 \%)(\mathrm{p}<0,05)$ (Tabla 1). El proceso inflamatorio agudo fue confirmado histológicamente en el $72 \%$ de los pacientes operados que tenían un carcinoma apendicular.

En tumores carcinoides la relación femenino: masculino es 1,2. Los tumores se localizaron en $1 / 3$ distal $69 \%$, $1 / 3$ medio $25 \%$ y $1 / 3$ proximal $6 \%$. El tamaño tumoral promedio fue 8,1 $\pm 4,9 \mathrm{~mm}$ y 4 casos $(6 \%)$ presentaron tamaño $\geq 2 \mathrm{~cm}$. La totalidad de carcinoides correspondió a tipo histológico clásico con infiltración intramucosa $2 \%$, submucosa $2 \%$, muscular propia $31 \%$, subserosa $34 \%$, serosa $10 \%$ y mesoapéndice $21 \%$. Se realizó hemicolectomía derecha en un $2^{\circ}$ tiempo en cinco pacientes, tres de los cuales presentaban infiltración tumoral en el mesoapéndice y dos en la serosa; no encontrándose lesión tumoral en la pieza quirúrgica correspondiente a la hemicolectomía. Todos ellos se encontraban vivos al momento del estudio (Tabla 1).

En adenocarcinomas la relación femenino: masculino fue 0,8. E1 50\% fueron adenocarcinomas mucinosos $\mathrm{y}$ $55 \%$ bien diferenciados. El nivel de infiltración tumoral fue $15 \%$ intramucosos, $10 \%$ submucosos, $25 \%$ muscular propia, 5\% subserosa, 30\% serosa y $15 \%$ mesoapéndice (Tabla 1). En 5 pacientes se efectuó apendicectomía con hemicolectomía derecha en un solo tiempo quirúrgico dado los hallazgos intra-operatorios y confirmación con biopsia contemporánea de la lesión tumoral; encontrándose metástasis ganglionar linfática y hepática en un caso, extensión tumoral a intestino delgado y grueso adyacente en un caso y no evidenciándose compromiso tumoral ganglionar linfático en tres pacientes. Se efectuó hemicolectomía derecha en $2^{\circ}$ tiempo quirúrgico en tres pacientes, encontrándose lesión neoplásica residual en la zona del ostium apendicular en 1 caso. 
DESCRIPCIÓN CLÍNICO-MORFOLÓGICA Y FACTORES PRONÓSTICOS EN CARCINOMAS DE APÉNDICE CECAL: ...

En adenocarcinoides la relación femenino: masculino fue 2 . Uno de ellos (33\%) presentó diferenciación mucinosa con células en anillo de sello. El nivel de infiltración tumoral fue 33\% muscular propia y $67 \%$ subserosa (Tabla 1 ). En ningún caso existió sospecha pre-operatoria ni compromiso de bordes quirúrgicos de sección. El 66\% (2 casos) se localizó en $1 / 3$ medio y uno (33\%) en $1 / 3$ distal y efectuó en todos ellos hemicolectomía derecha en $2^{\circ}$ tiempo quirúrgico, sin evidencias de lesión tumoral residual.

La mediana de seguimiento de la totalidad del grupo fue 76 meses (1 a 196 meses); el tiempo promedio de seguimiento para tumores carcinoides, adenocarcinomas y adenocarcinoides fue 80,83 y 106 meses respectivamente con una probabilidad de supervivencia actuarial global a 5 años para tumores carcinoides, adenocarcinomas y adenocarcinoides de $97 \%, 75 \%$ y $100 \%$ respectivamente $(p=0,009)$ (Figura 1).

La realización de hemicolectomía derecha en tumores carcinoides no modifica la supervivencia $(\mathrm{p}=$ 0,463 ); por el contrario en adenocarcinomas resulta ser una variable con diferencias estadísticamente significativas $(\mathrm{p}=0,04)$.

Al aplicar análisis divariados para supervivencia en la totalidad del grupo encontramos diferencias estadísticamente significativas en caso de sospecha pre-operatoria $(\mathrm{p}=0,009)$, tipo histológico $(\mathrm{p}=$ 0,009 ) (Figura 1) y compromiso tumoral de bordes quirúrgicos $(\mathrm{p}<0,001)$. Se observan diferencias estadísticamente significativas con tamaño tumoral $>2 \mathrm{~cm}$ en tumores carcinoides $(\mathrm{p}=0,001)$ (Figura 2) y según grado de diferenciación en adenocarcinomas $(\mathrm{p}<0,001)$ (Figura 3).

\section{Discusión}

Los carcinomas apendiculares son neoplasias poco frecuentes y están representados en su mayoría por lesiones con diferenciación neuroendocrina, los que por su parte presentan altas tasas de supervivencia cuando están confinados al apéndice, son pequeños $(<2 \mathrm{~cm})$ y no comprometen bordes quirúrgicos considerándose la apendicectomía como tratamiento en este grupo de pacientes ${ }^{1-12,23-25}$.

Al igual que en otros trabajos, la localización más frecuente de estos tumores es en el $1 / 3$ distal y en su mayoría lesiones menores de $10 \mathrm{~mm}$, confinadas al apéndice ${ }^{7,8}$.

Al igual que otras series publicadas, en nuestro estudio, adenocarcinomas y adenocarcinoides son lesiones poco frecuentes en el apéndice cecal; con una edad de presentación más tardía y diferente pronóstico que los carcinomas neuroendocrinos ${ }^{5,9-12}$.
Los tumores de localización apendicular generalmente son diagnosticados durante el examen anátomo-patológico de la pieza quirúrgica en pacientes operados por apendicitis aguda, siendo en una baja proporción sospechados pre-operatoriamente y en este caso asociados a mal pronóstico pues representan generalmente estadios avanzados de la enfermedad $^{1,3,13-17}$. En nuestra serie, 6 casos (7\%) fueron sospechados pre-operatoriamente (1 carcinoide y 5 adenocarcinomas); de los cuales 2 infiltraban hasta mesoapéndice, 1 serosa, 1 comprometía intestino delgado y grueso adyacente y 1 caso con metástasis hepática; encontrándose 2 fallecidos al momento del estudio.

Se describen tasas de supervivencia de $95 \%$ a 5 años para tumores carcinoides y $68 \%$ para adenocarcinomas $^{12,16,18,23,24}$, presentando los adenocarcinoides un comportamiento intermedio; aún cuando algunas series reportan una mayor agresividad y asociación con metástasis ováricas y peritoneales en adenocarcinoides ${ }^{25-28}$. En nuestro trabajo, al comparar la supervivencia del grupo de acuerdo al tipo histológico observamos tasas de supervivencia para carcinoides y adenocarcinomas similares a las publicadas $2,6,12,16,18,23,24,26,29$, sin embargo; en adenocarcinoides esta es superior a la reportada en distintas series $^{25-28}$; lo que podría ser explicado por el pequeño número de casos estudiados (3); junto con encontrarse estos tumores limitados al apéndice y en quienes se complementó el tratamiento con hemicolectomía derecha.

En 16 pacientes se realizó hemicolectomía derecha, (5 carcinoides, 8 adenocarcinomas y 3 adenocarcinoides); aún cuando las principales variables consideradas en este estudio para indicarla fueron nivel de infiltración tumoral, tipo histológico y compromiso de bordes quirúrgicos, no se realizó en todos los pacientes que cumplían criterios similares. Múltiples estudios $7,13,14,19,20,22,25,30,31$ señalan la necesidad de complementar con una hemicolectomía derecha en tumores carcinoides $>2 \mathrm{~cm}$ y/o con compromiso de mesoapéndice, sin embargo, trabajos recientes cuestionan la necesidad de realizarla, independiente del tamaño tumoral ${ }^{7,11,19,20}$. Al igual que lo publicado por otros autores ${ }^{7,19}$, en nuestra serie el tipo de cirugía no resultó una variable con diferencias estadísticamente significativas en la supervivencia de pacientes con carcinomas neuroendocrinos, lo que reafirma la controversia acerca del beneficio de realizar cirugías complementarias en sujetos con carcinomas neuroendocrinos. Por el contrario, para adenocarcinomas hay consenso en el beneficio de la hemicolectomía derecha en la supervivencia de estos pacientes ${ }^{1,16,18,32}$, tal cual observamos en nuestra serie al comparar este grupo, los cuales presentaron mejores tasas de supervivencia $(\mathrm{p}=0,04)$. 
En esta importante casuística de carcinomas apendiculares, el tipo histológico parece ser hasta ahora el principal indicador pronóstico y en la decisión de terapias complementarias, sin embargo, aún cuando observamos distinto impacto de la hemicolectomía derecha en la supervivencia de pacientes con carcinoides y adenocarcinomas apendiculares, estos resultados deben ser cuidadosamente evaluados teniendo en consideración las limitaciones propias del diseño de investigación con su respectivo nivel de evidencia.

Lo anteriormente expuesto plantea la necesidad de efectuar estudios prospectivos junto con la identificación de nuevos bio-marcadores que permitan al clínico decidir y ofrecer alternativas terapéuticas adecuadas a perfiles tumorales específicos.

\section{Referencias}

1. Connor S, Hanna G, Frizelle F. Appendiceal tumors: retrospective clinicopathologic analysis of appendix tumors from 7970 appendectomies. Dis Colon Rectum 1998; 41: 75-80.

2. McCusker M, Cote T, Clegg L, Sobin L. Primary malignant neoplasms of the appendix: a population based study from the surveillance, epidemiology and end-results program, 1973-1998. Cancer 2002; 94: 3307-3312.

3. Graham RP, Williams NP, West KA. Primary epithelial tumours of the appendix in a black population: a review of cases. World J Gastroenterol 2009; 15: 1472-1474.

4. Pŭrvanov P. Primary malignant appendiceal tumors. Khirurgiia 997; 50: 46-49.

5. Deans GT, Spence RA. Neoplastic lesions of the appendix. Br J Surg 1995; 82: 299-306.

6. McGory M, Maggard M, Kang H, O’Connell J, Ko C. Malignancies of the appendix: beyond case series reports. Dis Colon Rectum 2005; 48: 2264-2271.

7. Moertel CG, Dockerty MB, Judd ES. Carcinoid tumors of the vermiform appendix. Cancer 1968; 21: 270-278.

8. Lyss AP. Appendiceal malignancies. Semin Oncol 15: 129-137.

9. The WHO classification of tumours of the digestive system. Aaltonen LA, Hamilton SR. IARC, Lyon 2000.

10. Thomas RM, Sobin LH. Gastrointestinal cancer. Cancer 75: 154-170.

11. Fornaro R, Frascio M, Sticchi C, De Salvo L, Stabilini $\mathrm{C}$, Mandolfino F, et al. Appendectomy or right hemicolectomy in the treatment of appendiceal carcinoid tumors? Tumori 2007; 93: 587-590.

12. Park IJ, Yu CS, Kim HC, Kim JC. Clinical features and prognostic factors in primary adenocarcinoma of the appendix Korean J Gastroenterol 2004; 43: 29-34.

13. Yang WL, Yan CQ, Wang FJ, Wang HL. Diagnosis and surgical treatment of carcinoid tumors of the appendix in 64 patients. Zhonghua Zhong Liu Za Zhi; 2008; 30: 538-540.
14. Tchana-Sato V, Detry O, Polus M, Thiry A, Detroz B, Maweja S, et al. Carcinoid tumor of the appendix: a consecutive series from 1237 appendectomies. World J Gastroenterol 2006; 12: 6699-6701.

15. Chen HT, Lee YT, Chou AS, Wu YK, Yin WY, Lee MC, et al. Primary appendiceal malignancy: a clinicopathologic study. Kaohsiung J Med Sci 2006; 22: 618-625.

16. Cortina R, McCormick J, Kolm P, Perry RR. Management and prognosis of adenocarcinoma of the appendix. Dis Colon Rectum 1995; 38: 848-852.

17. In't Hof KH, van der Wal HC, Kazemier G, Lange JF. Carcinoid tumour of the appendix: an analysis of 1.485 consecutive emergency appendectomies. J Gastrointest Surg 2008; 12: 1436-1438. Epub 2008 Jun 3.

18. Nitecki S, Wolff B, Schlinkert R, Sarr M. The natural history of surgically treated primary adenocarcinomas of the appendix. Ann Surg 1994; 219: 51-57.

19. Bamboat $Z$, Berger D. Is right hemicolectomy for 2.0 $\mathrm{cm}$ appendiceal carcinoids justified? Arch Surg 2006; 141: 349-352.

20. Safioleas MC, Moulakakis KG, Kontzoglou K, Stamoulis J, Nikou GC, Toubanakis C, et al. Carcinoid tumors of the appendix. Prognostic factors and evaluation of indications for right hemicolectomy. Hepatogastroenterology 2005; 52: 123-127.

21. Rutledge RH, Alexander JW. Primary appendiceal malignancies: rare but important. Surgery 1992; 111: 244-250.

22. Thompson GB, Van-Heerden JA, Martin-JK J, Schutt AJ, Ilstrup DM, Carney JA. Carcinoid tumors of the gastrointestinal tract: presentation, management, and prognosis. Surgery 1985; 98: 1054-1063.

23. MacGillivray DC, Heaton RB, Rushin JM, Cruess DF. Distant metastasis from a carcinoid tumor of the appendix less than one centimeter in size. Surgery 1992; 111: 466-471.

24. Modlin IM, Sandor A. An analysis of 8305 cases of carcinoid tumors. Cancer 1997; 79: 813-829.

25. You J, Xu L, Zheng GY, Qiu JF. Diagnosis, treatment, and prognosis of primary appendiceal tumors: analysis of 37 cases. Zhonghua Yi Xue Za Zhi 2008; 88: 19091911.

26. Bucher P, Gervaz P, Ris F, Oulhaci W, Egger J, Morel P. Surgical treatment of appendiceal adenocarcinoid (goblet cell carcinoid). World J Surg 2005; 29: 1436-1439.

27. Pérez-Alonso P, Blanco Álvarez J, Aso Manso S. Tumor adenocarcinoide apendicular (subtipo de células caliciformes). Rev Esp Patol 2004; 37: 337-338.

28. Cerdán G, Artigas V, Llauradó JM, Rodríguez M, Sancho FJ, Moral A, y cols. Adenocarcinoide apendicular: factores pronósticos y actitud terapéutica. Rev Esp Enferm Dig 2001; 93: 190-1.

29. Ito H, Osteen R, Bleday R, Zinner M, Ashley S, Whang E. Appendiceal adenocarcinoma: long-term outcomes after surgical therapy. Dis Colon Rectum 2004; 47: 474480 . 
DESCRIPCIÓN CLÍNICO-MORFOLÓGICA Y FACTORES PRONÓSTICOS EN CARCINOMAS DE APÉNDICE CECAL: ...

30. Moertel C, Weiland L, Nagorney D, Dockerty M. Carcinoid tumor of the appendix: treatment and prognosis. N Engl J Med 1987; 317: 1699-1701.

31. Gouzi JL, Laigneau P, Delalande J, Flamant Y, Bloom E, Oberlin $\mathrm{P}$, et al. Indications for right hemicolectomy in carcinoid tumors of the appendix. The French Associations for Surgical Research. Surg Gynecol Obstet 1993; 176: 543-547.

32. Lenriot J, Huguier M. Adenocarcinoma of the appendix. Am J Surg 1988; 155: 470-475. 